\title{
Targeting the integrated networks of aggresome formation, proteasome, and autophagy potentiates ER stress-mediated cell death in multiple myeloma cells
}

\author{
SHOTA MORIYA $^{1}$, SEIICHIRO KOMATSU ${ }^{2}$, KAHO YAMASAKI $^{1}$, YUSUKE KAWAI ${ }^{1}$, \\ HIROKO KOKUBA $^{3}$, AYAKO HIROTA ${ }^{1}$, XIAO-FANG CHE $^{1}$, MASATO INAZU ${ }^{4}$, \\ AKIHIKO GOTOH $^{5}$, MASAKI HIRAMOTO ${ }^{1}$ and KEISUKE MIYAZAWA ${ }^{1}$ \\ Departments of ${ }^{1}$ Biochemistry and ${ }^{2}$ Breast Oncology, ${ }^{3}$ Laboratory of Electron Microscopy, ${ }^{4}$ Institute of \\ Medical Science, Tokyo Medical University; ${ }^{5}$ Department of Hematology, Juntendo University, Tokyo, Japan
}

Received September 22, 2014; Accepted October 30, 2014

DOI: 10.3892/ijo.2014.2773

\begin{abstract}
The inhibitory effects of macrolide antibiotics including clarithromycin (CAM) on autophagy flux have been reported. Although a macrolide antibiotic exhibits no cytotoxicity, its combination with bortezomib (BZ), a proteasome inhibitor, for the simultaneous blocking of the ubiquitin (Ub)-proteasome and autophagy-lysosome pathways leads to enhanced multiple myeloma (MM) cell apoptosis induction via stress overloading of the endoplasmic reticulum (ER). As misfolded protein cargo is recruited by histone deacetylase 6 (HDAC6) to dynein motors for aggresome transport, serving to sequester misfolded proteins, we further investigated the cellular effects of targeting proteolytic pathways and aggresome formation concomitantly in MM cells. Pronounced apoptosis was induced by the combination of vorinostat [suberoylanilide hydroxamic acid (SAHA); potently inhibits HDAC6] with CAM and BZ compared with each reagent or a 2-reagent combination. CAM/BZ treatment induced vimentin positive-aggresome formation along with the accumulation of autolysosomes in the perinuclear region, whereas they were inhibited in the presence of SAHA. The SAHA/CAM/BZ combination treatment maximally upregulated genes related to ER stress including C/EBP homologous protein (CHOP). Similarly to MM cell lines, enhanced cytotoxicity with CHOP upregulation following SAHA/CAM/BZ treatment was shown by a wild-type murine embryonic fibroblast (MEF) cell line; however, a CHOP-deficient MEF cell line almost completely canceled this pronounced cytotoxicity. Knockdown of HDAC6 with siRNA exhibited further enhanced CAM/BZ-induced
\end{abstract}

Correspondence to: Dr Keisuke Miyazawa, Department of Biochemistry, Tokyo Medical University, 6-1-1 Shinjuku, Shinjuku-ku, Tokyo 160-8402, Japan

E-mail: miyazawa@tokyo-med.ac.jp

Key words: multiple myeloma, aggresome, proteasome, autophagy, ER stress cytotoxicity and CHOP induction along with the cancellation of aggresome formation. Targeting the integrated networks of aggresome, proteasome, and autophagy is suggested to induce efficient ER stress-mediated apoptosis in MM cells.

\section{Introduction}

Multiple myeloma (MM) is a refractory hematopoietic malignancy showing clonal plasma cell accumulation. A major breakthrough in MM treatment has been the introduction of the first-in-class proteasome inhibitor bortezomib (BZ) $(1,2)$. Moreover, the treatment of relapsed and refractory MM is now possible with carfilzomib, a second-generation proteasome inhibitor, and immunomodulatory agents. This has offered new alternatives for vulnerable patients $(3,4)$. However, patients with relapsed and refractory MM are still urgent issues and require treatment combinations $(5,6)$. Increasing lines of evidence indicate that proteasome inhibition induces misfolded protein accumulation in the endoplasmic reticulum (ER) $(7,8)$. This evokes ER stress followed by the unfolded protein response (UPR) $(9,10)$. UPR mainly functions: i) to decrease protein entry into the ER by suppressing translational rate; and ii) to increase the folding capacity of the ER via chaperon protein translational activations. Following incorrect folding in the ER, proteins are retro-translocated for degradation in the cytoplasm via the ubiquitin (Ub)-proteasome pathway [i.e., ER-associated degradation (ERAD)]. A failure in all the adaptation strategies triggers apoptosis and induces C/EBP homologous protein (CHOP) (GADD153), a pro-apoptotic transcription factor and other pathways (7-10). Since MM is characterized by the uncontrolled cell growth of monoclonal antibody (mAb)-producing plasma cells, production of large quantities of unfolded or misfolded immunoglobulin triggers ER stress. Thus, therapeutic manipulation of the UPR pathway appears to disrupt cellular mechanisms for processing high protein loads and cellular stress, and further leads to death of MM cells.

Macroautophagy (hereafter, 'autophagy') occurs when cellular proteins and organelles (e.g., ER) are enveloped in an autophagosome and degraded in lysosomes by lysosomal 
hydrolases $(11,12)$. Although autophagy is considered a bulk non-selective degradation of long-lived proteins and organelles, recent reports revealed the selective degradation pathway of ubiquitinated protein via autophagy using docking proteins (e.g., p62) and related proteins (e.g., NBR1), having both a microtubule-associated protein 1 light chain 3 (LC3)-interacting region and a Ub-associated domain $(13,14)$. Thus, overflowed ubiquitinated proteins are bound to p62 and subsequently engulfed into an autophagosome via the LC3-interacting region in p62. This indicates that autophagy also acts as a compensatory degradation system when the proteasome system is impaired (14). We previously reported that the inhibition of autophagy using the autophagy inhibitor bafilomycin $\mathrm{A}_{1}$ (BAF) enhanced BZ-induced apoptosis by burdening ER stress in MM cell lines (15). We also reported that macrolide antibiotics [e.g., clarithromycin (CAM) and azithromycin (AZM)] attenuated or blocked autophagy flux, possibly mediated through the inhibition of the lysosomal function, and that ER stress loading was enhanced by both the BZ-induced inhibition of the Ub-proteasome system and the CAM- or AZM-induced inhibition of the autophagy-lysosome system. This is followed by CHOP transcriptional activation and the induction of apoptosis in $\mathrm{MM}$ and breast cancer cells $(16,17)$. Therefore, concomitant blocking of proteasome and autophagy appears to be a promising combination therapy.

Moreover, misfolded/unfolded proteins are sequestrated into aggregates and transported. They are then discarded from the cytoplasm by dynein motors through the microtubule network to the aggresome (18). The class II histone deacetylase 6 (HDAC6), which is a microtubule-associated deacetylase and an aggresome component, has the capacity to bind both polyubiquitinated misfolded proteins and dynein motors (19). By acting as an adaptor between ubiquitinated protein aggregates and dynein, HDAC6 enables aggregated protein loading onto the dynein motor protein complex (20). Thus, in the formation of aggresome at the microtubule-organizing center (MTOC), HDAC6, dynein, and polyubiquitinated proteins functionally interact with each other $(19,20)$. If HDAC6 is lacking, cells will not be able to clear cytoplasmic unfolded protein aggregates; cells cannot appropriately form aggresomes; and cells become hypersensitive to unfolded protein accumulation (19). Therefore, HDAC6 appears to be another critical factor in the management of unfolded protein-induced stress at the cellular level. Moreover, some parts of the aggresome are reported to be degraded via the autophagy-lysosome system $(21,22)$. A recent study also revealed that p62 regulates the accumulation and autophagic clearance of protein aggregates by directly binding to HDAC6, and this interaction appears to regulate HDAC6 activity (23).

All these lines of evidence suggest the integrated intracellular networks of proteasome, autophagy, and aggresome for unfolded protein processing. ER stress loading may be further enhanced by targeting both intracellular proteolytic pathways and aggresome formation (24). To prove this hypothesis aimed for clinical application, we intentionally attempted to use well-approved drugs such as CAM, BZ, and vorinostat [suberoylanilide hydroxamic acid (SAHA)], an orally bioavailable inhibitor of HDAC with a half maximal inhibitory concentration $\left(\mathrm{IC}_{50}\right)$ of $37 \mathrm{nM}$ and which has passed the assessment of the Food and Drug Administration for cutaneous T-cell lymphoma treatment $(25,26)$. In the present investigation, we clearly demonstrated that the SAHA/CAM/BZ combination treatment induced marked ER stress-mediated MM cell death. This provides a promising treatment for MM patients in the form of 'ER stress-loading therapy'.

\section{Materials and methods}

Reagents. BZ was purchased from Selleck Chemicals (Houston, TX, USA). CAM was purchased from Tokyo Chemical Industry Co., Ltd. (Tokyo, Japan), SAHA was from Cayman Chemical Co. (Ann Arbor, MI, USA), and tubacin was from Sigma-Aldrich (St. Louis, MO, USA). BZ, SAHA and tubacin were dissolved in dimethyl sulfoxide to make stock solutions at concentrations of 1,10 and $1 \mathrm{mM}$, respectively. CAM was dissolved in ethanol to prepare stock solutions of $5 \mathrm{mg} / \mathrm{ml}$.

Cell lines and culture conditions. For this study, the MM cell lines IM-9 and RPMI-8226, and the lung carcinoma cell line H226 were obtained from the American Type Culture Collection (ATCC) (Manassas, VA, USA). The human MM cell line KMS-12-PE was obtained from the Japanese Collection of Research Bioresources (JCRB) (Osaka, Japan). A CHOP ${ }^{-/-}$murine embryonic fibroblast (MEF) cell line (CHOP-KO-DR) established from a 13.5-day-old $\mathrm{CHOP}^{-/-}$ mouse embryo by SV-40 immortalization and a $\mathrm{CHOP}^{+/+} \mathrm{MEF}$ cell line (DR-wild-type) established by SV-40 immortalization as a control cell line for CHOP-KO-DR were also obtained from the ATCC. These authorized cell lines were expanded and frozen in aliquots within 1 month after obtaining from the cell banks. Each aliquot was thawed and the cells were used for the experiments within 2 months after thawing. IM-9, H226, RPMI-8226, and KMS-12-PE cells were cultured in RPMI-1640 medium (Sigma-Aldrich) supplemented with 10\% fetal bovine serum (FBS) (Biowest SAS, Nuaillé, France), $2 \mathrm{mM}$ L-glutamine, penicillin $(100 \mathrm{U} / \mathrm{ml})$, and streptomycin $(100 \mu \mathrm{g} / \mathrm{ml})$ (Wako Pure Chemicals Industries, Tokyo, Japan). CHOP-KO-DR and DR-wild-type cells were maintained in Dulbecco's modified Eagle's medium (Sigma-Aldrich) supplemented with $10 \%$ FBS, 2 mM L-glutamine, penicillin (100 U/ml), and streptomycin $(100 \mu \mathrm{g} / \mathrm{ml})$. All cell lines were cultured in a humidified incubator containing $5 \% \mathrm{CO}_{2}$ and $95 \%$ air at $37^{\circ} \mathrm{C}$.

Assessment of viable number of cells. The number of viable cells was assessed using CellTiter-Blue Cell Viability Assay kit (Promega Corp., Madison, WI, USA) according to the manufacturer's instructions as previously described in detail (16).

Immunoblotting. Immunoblotting was performed as previously described $(15,16)$. In brief, cells were lysed with RIPA lysis buffer supplemented with a protease and phosphatase inhibitor cocktail (both from Nacalai Tesque, Kyoto, Japan). Cellular proteins were quantified using a DC Protein Assay kit (Bio-Rad, Hercules, CA, USA). Equal amounts of proteins were loaded onto the gels, separated by sodium dodecyl sulfate polyacrylamide gel electrophoresis (SDS-PAGE), and transferred onto Immobilon-P membrane (Millipore, Billerica, MA, USA). The membranes were probed with primary antibodies (Abs) such as anti-acetylated- $\alpha$-tubulin (6-11B-1) 

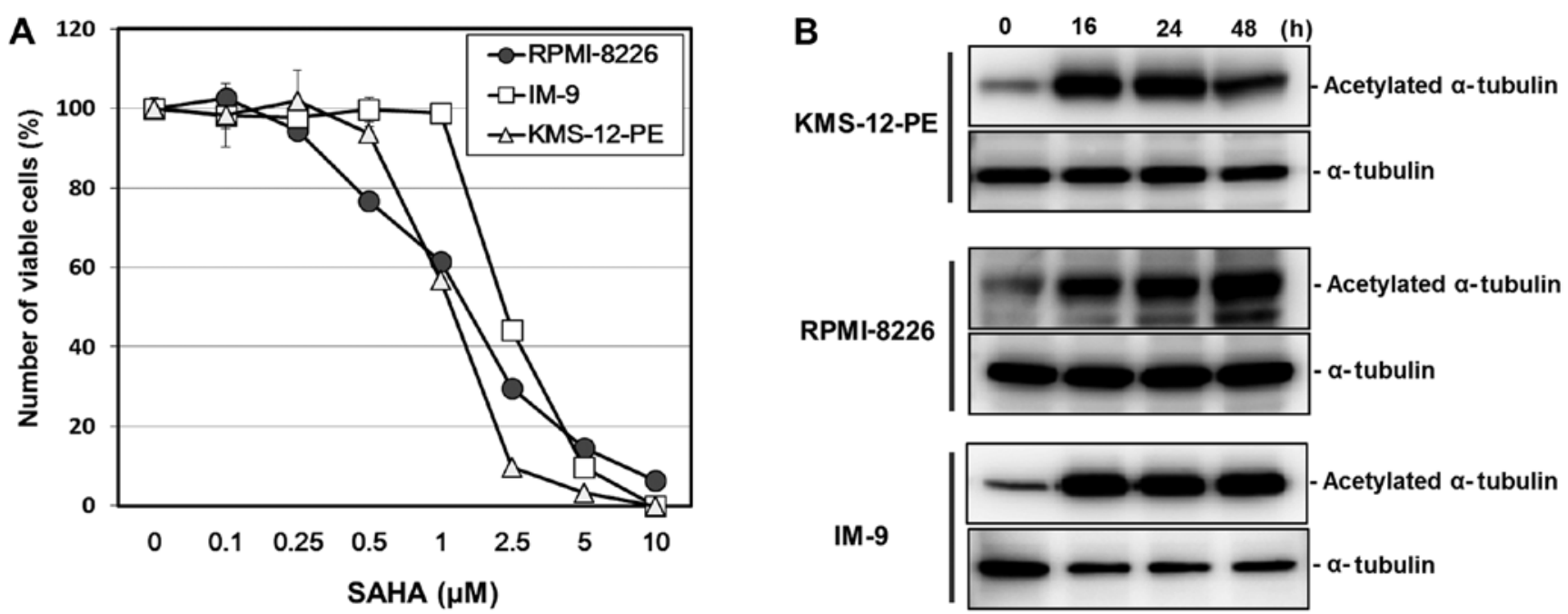

Figure 1. Cell growth inhibition and $\alpha$-tubulin acetylation after treatment with suberoylanilide hydroxamic acid (SAHA) in myeloma cell lines. (A) The myeloma cells KMS-12-PE, RPMI-8226, and IM-9 were cultured in the presence of SAHA at various concentrations for $48 \mathrm{~h}$. Cell growth inhibition was assessed as described in Materials and methods. (B) After treatment with SAHA (at $0.5 \mu \mathrm{M}$ in KMS-12-PE, RPMI-8226, and at 1.0 $\mu \mathrm{M}$ in IM-9) for 16-48 $\mathrm{h}$ cellular proteins were lysed, separated by $11.25 \%$ sodium dodecyl sulfate polyacrylamide gel electrophoresis (SDS-PAGE), and immunoblotted using a specific monoclonal antibody $(\mathrm{mAb})$ against the acetylated $\alpha$-tubulin. Immunoblotting with anti- $\alpha$-tubulin $\mathrm{mAb}$ was performed as an internal control.

$\mathrm{mAb}$, anti- $\alpha$-tubulin (B-7) mAb, anti-GAPDH (6C5) $\mathrm{mAb}$ anti-HDAC6 (H-300) Ab, and anti-Ub (P4D1) mAb, which were all purchased from Santa Cruz Biotechnology, Inc. (Santa Cruz, CA, USA) and anti-PARP Ab (Cell Signaling Technology, Inc., Danvers, MA, USA). Immunoreactive proteins were detected with horseradish peroxidase-conjugated secondary Abs (Cell Signaling Technology, Inc.) and an enhanced chemiluminescence reagent (Millipore). Densitometry was performed using a Molecular Imager ChemiDoc XRS System (Bio-Rad).

RNA interference. For the gene silencing of HDAC6 in RPMI-8226 and H226 cells, HDAC6 siRNA and control siRNA were purchased from Life Technologies (Grand Island, NY, USA) and whose sequences are described as follows: HDAC6 sense, CCAGCACAGUCUUAUGGAUGGCUAU and antisense, AUAGCCAUCCAUAAGACUGUGCUGG. siRNAs were diluted to a final concentration of $33 \mathrm{nM}$ in Opti-MEM I (Life Technologies). Transfection was performed with the cells at $40 \%$ confluency using Lipofectamine RNAiMAX transfection reagent (Life Technologies) according to the manufacturer's instructions. Knockdown efficiency was assessed by immunoblotting.

Gene expression analysis. Total RNA was isolated from cell pellets using Isogen (Wako Pure Chemicals Industries) and genomic DNA was removed using RQ1 RNase-Free DNase (Promega Corp.) at $37^{\circ} \mathrm{C}$ for $30 \mathrm{~min}$, followed by extraction with phenol chloroform and ethanol precipitation. Reverse-transcription using a PrimeScript RT Master Mix (Takara Bio, Inc., Shiga, Japan) was performed according to the manufacturer's instructions. Real-time polymerase chain reaction (PCR) was performed on $3 \mathrm{ng}$ of cDNA using validated SYBR-Green gene expression assays for human ER stress-related genes (CHOP, BAX, BIM, DR5, GADD34 and GRP78) in combination with SYBR Premix Ex Taq II (Takara Bio, Inc.). The sequences of primers and reaction conditions were previously described (16). Quantitative real-time
PCR was performed in duplicates in a Thermal Cycler Dice Real-Time System TP800 (Takara Bio, Inc.). The data were analyzed using Thermal Cycler Dice Real-Time System Software version 5.00 (Takara Bio, Inc.), and the comparative $\mathrm{Ct}$ method $\left(2^{-\Delta \Delta \mathrm{Ct}}\right)$ was used for the relative quantification of gene expression. The data of real-time PCR products were standardized to GAPDH as an internal control. To confirm the specific amplification of target genes, each gene product was further separated by using $1.5 \%$ agarose gel after real-time PCR to detect a single band at the theoretical product size, as well as by analysis of the dissociation curve for detecting a single peak.

Immunocytochemistry and confocal microscopy. Cells were spread on slide glasses using Cytospin 4 Centrifuge (Thermo Fisher Scientific, Inc., Rockford, IL, USA) to make slide glass preparations. Cells were fixed for $20 \mathrm{~min}$ in ice-cold methanol and permeabilized with $0.1 \%$ Triton X-100 for $20 \mathrm{~min}$, followed by blocking with $2 \%$ bovine serum albumin in TBST $(25 \mathrm{mM}$ Tris, $137 \mathrm{mM} \mathrm{NaCl}, 2.7 \mathrm{mM} \mathrm{KCl}, 0.05 \%$ Tween-20, pH 7.4) for $1 \mathrm{~h}$. Cells were immunostained with primary Abs such as mouse anti-vimentin (V9) mAb, mouse anti-Ub mAb, and lysosomal-associated membrane protein-1 (LAMP-1) (H4A3) mAbs (all from Santa Cruz Biotechnology, Inc.). The secondary Abs used for fluorescence detection were Alexa Fluor ${ }^{\circledR} 488 \mathrm{~F}\left(\mathrm{ab}^{\prime}\right) 2$ fragment of goat anti-mouse IgG $(\mathrm{H}+\mathrm{L}) \mathrm{Ab}$ (Life Technologies). Nuclei were stained with 4',6-diamidino-2-phenylindole (DAPI) (Wako Pure Chemicals Industries). Slides were mounted with SlowFade Gold antifade reagent (Life Technologies). Analysis by confocal microscopy was performed using the confocal laser scanning fluorescence microscope FV10i-DOC (Olympus Corp., Tokyo, Japan).

Assessment of aggresome by fractionation of detergent-soluble and-insoluble proteins. Cells were lysed with Triton X-100 lysis buffer (10 mM Tris- $\mathrm{HCl}, 150 \mathrm{mM} \mathrm{NaCl}, 2 \%$ Triton X-100, $\mathrm{pH} 7.8$ ) supplemented with a protease inhibitor cocktail (Nacalai 
A
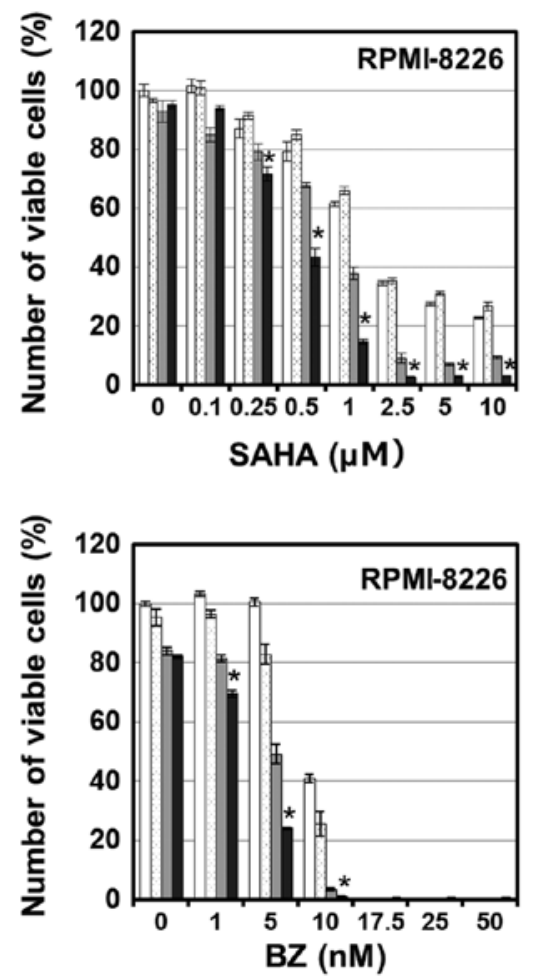
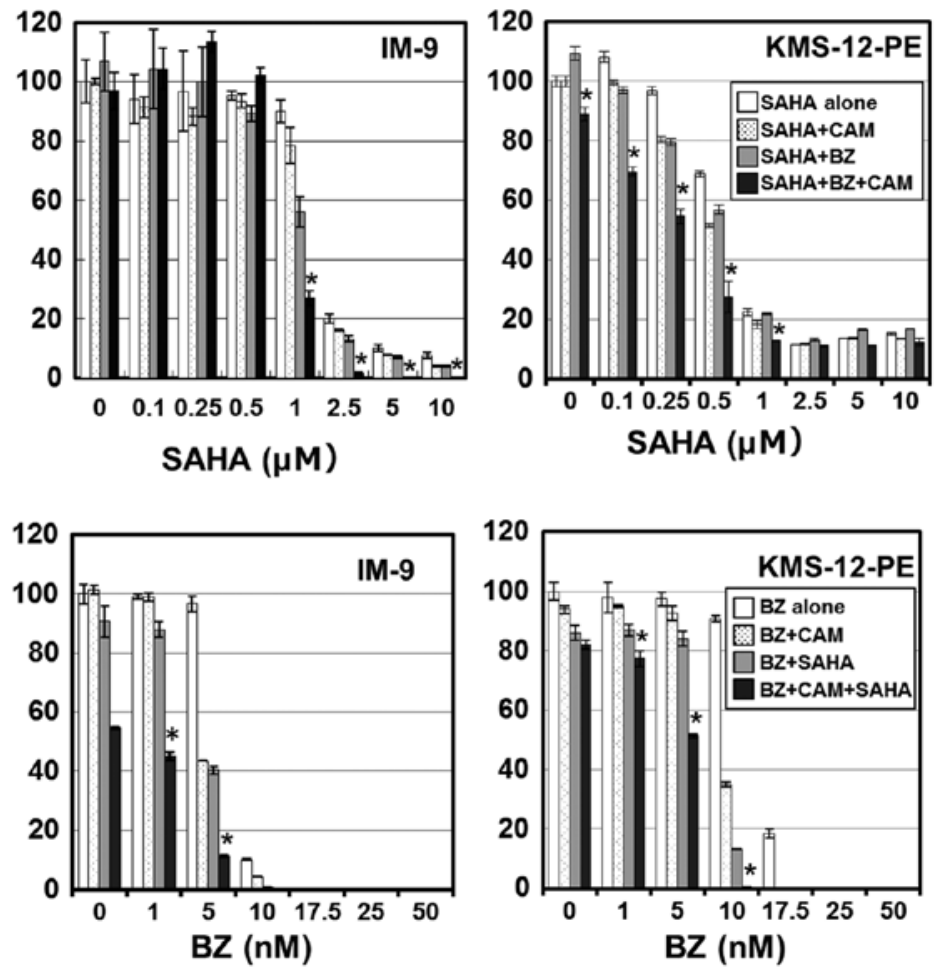

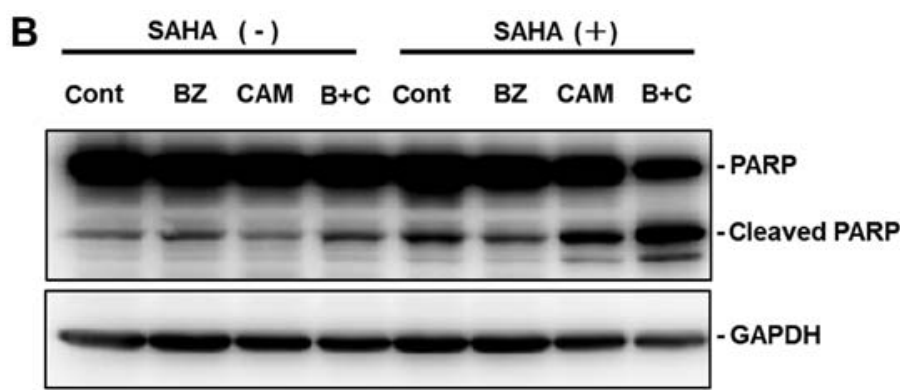

Figure 2. Cell growth inhibition after treatment with suberoylanilide hydroxamic acid (SAHA), bortezomib (BZ), and clarithromycin (CAM) in myeloma cell lines. (A) Upper panels: myeloma cell lines RPMI-8226, IM-9, and KMS-12-PE were cultured in the presence of various concentrations of SAHA with/without either BZ $(5 \mathrm{nM})$ or CAM $(50 \mu \mathrm{g} / \mathrm{ml})$ for $48 \mathrm{~h}$. Lower panels: myeloma cell lines were cultured in the presence of various concentrations of BZ with/without either SAHA (at $0.5 \mu \mathrm{M}$ in KMS-12-PE, RPMI-8226, and at $1.0 \mu \mathrm{M}$ in IM-9) or CAM (50 $\mu \mathrm{g} / \mathrm{ml})$ for $48 \mathrm{~h}$. Viable cell numbers were assessed as described in Materials and methods. ${ }^{*}<0.05$ SAHA/BZ/CAM vs. SAHA/BZ and SAHA/CAM and BZ/CAM. (B) IM-9 cells were treated with either BZ (5 nM) or CAM $(50 \mu \mathrm{g} / \mathrm{ml})$ in the presence or absence or SAHA $(1 \mu \mathrm{M})$ for $48 \mathrm{~h}$. Cellular proteins were separated by $11.25 \%$ sodium dodecyl sulfate polyacrylamide gel electrophoresis (SDS-PAGE), and immunoblotted with anti-PARP monoclonal antibody (mAb). Immunoblotting with GAPDH mAb was performed as an internal control for protein loading.

Tesque). The lysates were centrifuged at $12,000 \mathrm{~g}$ for $30 \mathrm{~min}$ at $4^{\circ} \mathrm{C}$. The supernatant was then collected as the soluble fraction. The pellets (which contain the insoluble protein) were then resuspended in sodium dodecyl sulfate (SDS) lysis buffer (10 mM Tris- $\mathrm{HCl}, 150 \mathrm{mM} \mathrm{NaCl}, 2 \%$ SDS, $\mathrm{pH}$ 7.8) and sonicated for $30 \mathrm{sec}$ with a tip sonicator VP-5S (Taitec, Saitama, Japan) to prepare the insoluble fraction. Equal volumes of each pellet and supernatants were boiled for $5 \mathrm{~min}$ in SDS-PAGE sample buffer (125 mM Tris- $\mathrm{HCl}, 4 \%$ SDS, $20 \%$ glycerol, $0.002 \%$ BPB, pH 6.8) and analyzed by SDS-PAGE.

Electron microscopy. Cells were fixed with $2.5 \%$ glutaraldehyde in $0.1 \mathrm{M}$ phosphate buffer ( $\mathrm{pH} 7.4)$ for $1 \mathrm{~h}$. The samples were further fixed in $1 \%$ osmium tetroxide for $1 \mathrm{~h}$, dehydrated in graded ethanol (30-100\%), and embedded in Quetol 812 epoxy resin (Nisshin EM Co., Ltd., Tokyo, Japan). Ultrathin sections were cut with an Ultracut J microtome (Reichert-Jung, Vienna, Austria). The sections were stained with lead nitrate and uranium acetate, and subjected to electron microscopic analysis using the scanning electron microscope JEM-1200 EXII (JEOL, Tokyo, Japan).

Statistical analysis. All data are expressed as mean \pm SD. Statistical analysis was performed using Mann-Whitney U test (two-tailed).

\section{Results}

SAHA, BZ, and CAM combination treatment potently enhanced MM cell apoptosis. Treatment with SAHA for $48 \mathrm{~h}$ resulted in a dose-dependent inhibition of cellular growth in all MM cell lines (Fig. 1A). The $\mathrm{IC}_{50}$ was $1.2 \mu \mathrm{M}$ in KMS-12-PE, $1.5 \mu \mathrm{M}$ in 

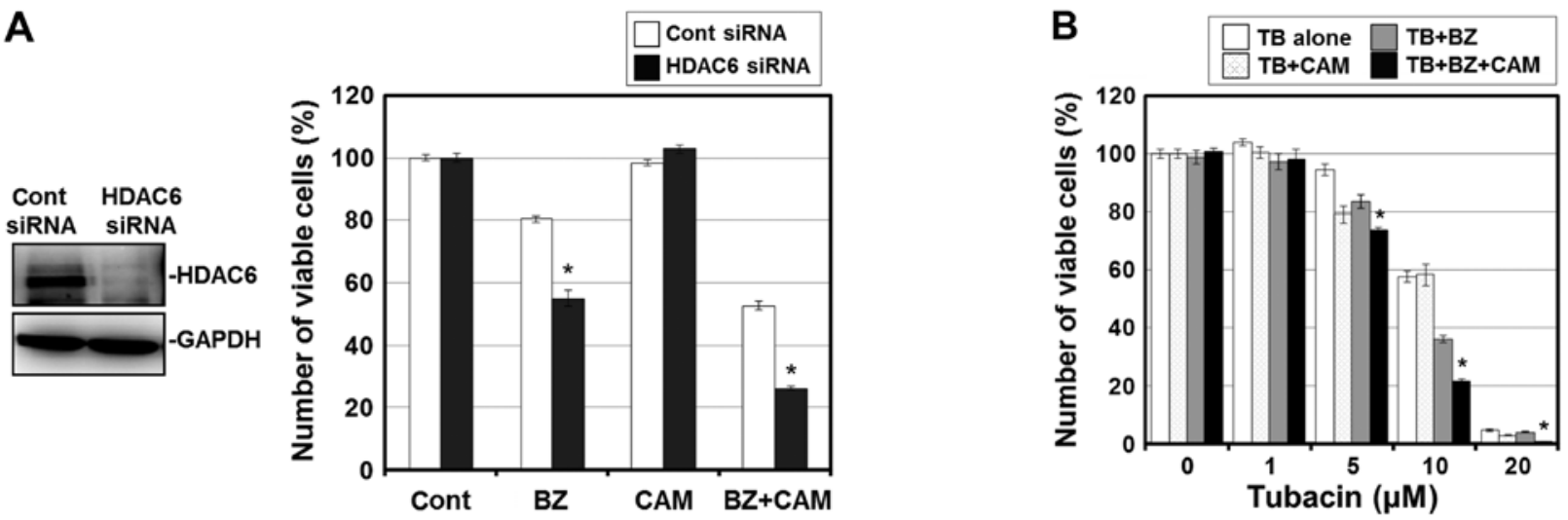

Figure 3. Effects of histone deacetylase 6 (HDAC6) inhibition on myeloma cell growth. (A) Suppression of HDAC6 expression using HDAC6 siRNA: RPMI-8226 cells were pre-treated with either HDAC6 siRNA or control siRNA for $48 \mathrm{~h}$. Subsequently, the cells were cultured in the presence or absence of bortezomib (BZ) $(10 \mathrm{nM})$ with/without clarithromycin (CAM) $(50 \mu \mathrm{g} / \mathrm{ml})$ for $48 \mathrm{~h}$. "P<0.05 cont siRNA vs. HDAC6 siRNA. (B) Enzymatic inhibition of HDAC6 with a specific HDAC6 inhibitor, tubacin: RPMI-8226 cells were treated with tubacin at indicated concentrations in the presence or absence of either BZ $(5 \mathrm{nM})$ or CAM $(50 \mu \mathrm{g} / \mathrm{ml})$ for $48 \mathrm{~h}$. Viable cell numbers were assessed as described in Materials and methods. ${ }^{*} \mathrm{P}<0.05 \mathrm{~TB} / \mathrm{BZ} / \mathrm{CAM} v \mathrm{vs}$. TB/BZ and TB/CAM.

RPMI-8226, and $2.1 \mu \mathrm{M}$ in IM-9 cells. SAHA-treated MM cells exhibited apoptotic morphologic features such as fragmentation of the nucleus and formation of an apoptotic body along with cleavage of PARP and caspase-3 (data not shown). The regulation of the stability and function of a microtubule has been associated with $\alpha$-tubulin reversible acetylation, and HDAC6 functions as $\alpha$-tubulin deacetylase (27). Immunoblotting using a specific $\mathrm{Ab}$ for the acetylated $\alpha$-tubulin revealed that, in response to SAHA (at $0.5 \mu \mathrm{M}$ for KMS-12-PE and RPMI-8226, at $1 \mu \mathrm{M}$ for IM-9), the acetylation of $\alpha$-tubulin was detectable within $16 \mathrm{~h}$ and persisted for at least $48 \mathrm{~h}$ in all three cell lines tested (Fig. 1B). In our previous studies, autophagy flux was shown to be blocked by CAM, and that the BZ and CAM combination treatment resulted in ER-stress overloading followed by enhanced induction of apoptosis in MM and breast cancer cells $(16,17)$. Since HDAC6 has been implicated in aggresome formation that results in sequestering overabundant intracellular unfolded proteins (18), we examined whether the SAHA plus BZ and/or CAM combination treatment further increased cytotoxicity via ER stress loading. As shown in Fig. 2A, the combined treatment with SAHA/BZ, but not with SAHA/CAM, potentiated cell growth inhibition. Notably, although CAM treatment alone at $50 \mu \mathrm{g} / \mathrm{ml}$ did not inhibit the growth of cells, the combined SAHA/BZ/CAM treatment showed a clearly pronounced cytotoxicity compared with the SAHA/BZ or BZ/CAM treatment in all MM cell lines. This enhanced cytotoxicity was mediated through apoptosis induction since the pronounced expression of the cleaved PARP was observed in response to the suboptimal concentrations of the SAHA/BZ/CAM combination in IM-9 cells (Fig. 2B).

To confirm that this enhanced cytotoxicity is mediated through HDAC6 inhibition, we next attempted to knock down HDAC6 with siRNA. Pre-treatment with HDAC6 siRNA effectively suppressed the expression level of HDAC6 in RPMI-8226 cells. Under this condition, the cell growth inhibition by the BZ/CAM treatment was pronounced compared with the cells pre-treated with control siRNA. In addition, tubacin, a specific HDAC6 inhibitor, reproduced the pronounced cytotoxicity when combined with BZ and BZ/CAM (Fig. 3A and B) (28). These results suggest that HDAC6 inhibition appears to be involved in the enhancement of cytotoxicity induced by the addition of SAHA to BZ/CAM-containing cell culture medium.

SAHA suppressed the aggresome formation induced by simultaneous inhibition of proteasome and autophagy in MM cells. To further confirm that the pronounced cytotoxicity by SAHA shown in Fig. 2A was due to the inhibition of HDAC6 activity involved in aggresome formation, we assessed aggresome formation in the presence or absence of SAHA. In RPMI-8226 cells, immunocytochemistry using anti-vimentin $\mathrm{mAb}$ showed that the combined treatment with BZ/CAM induced a dense deposit of vimentin in the perinuclear region, which is a well-known marker for aggresome (29). This vimentin deposit was clearly suppressed in the presence of SAHA (Fig. 4A). However, since the MM cell lines including RPMI-8226 were very sensitive to the SAHA/BZ/CAM treatment, in which apoptosis was induced within $18 \mathrm{~h}$ of exposure to these reagents (data not shown), precise analysis of aggresome formation was practically difficult using MM cells. We therefore used the lung squamous carcinoma cell line H226 which is less sensitive, but shows pronounced cytotoxicity in response to the SAHA/BZ/CAM combination similarly to MM cell lines (Fig. 4B). As shown in Fig. 4C, the prominent vimentin deposit in the perinuclear region was observed after combined treatment with BZ/CAM for $24 \mathrm{~h}$. The vimentin deposit with high fluorescent intensity was evidently suppressed in the presence of SAHA, indicating that SAHA inhibits BZ/CAM-induced aggresome formation (Fig. 4C). Similarly to SAHA, treatment with HDAC6 siRNA suppressed the vimentin condensation in the perinuclear region in $\mathrm{H} 226$ cells (Fig. 4D-F).

In addition, immunocytochemistry using anti-Ub $\mathrm{Ab}$ exhibited the clustering of Ub-positive dots in the perinuclear region by BZ/CAM, whereas they were dispersed in the presence of SAHA in H226 cells (data not shown). As an alternative assessment of aggresome, we treated KMS-12-PE with BZ, $\mathrm{CAM}$, or BZ/CAM in the presence or absence of SAHA. The cells were then treated with lysis buffer containing $2 \%$ 

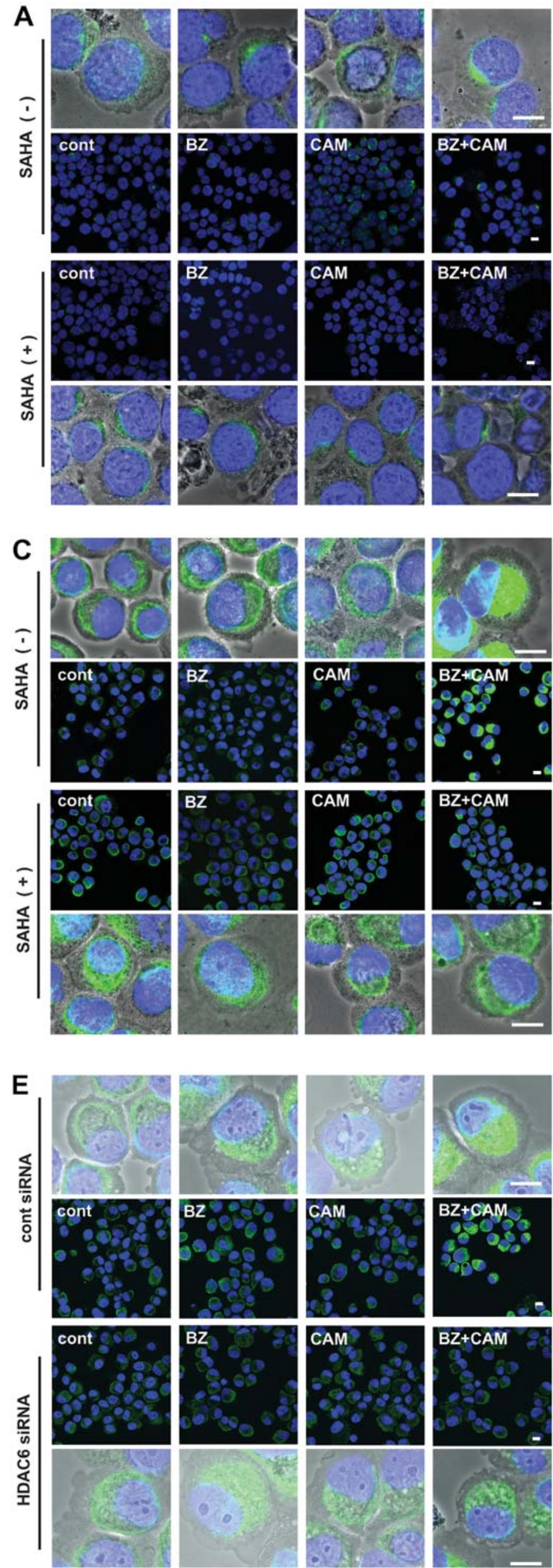
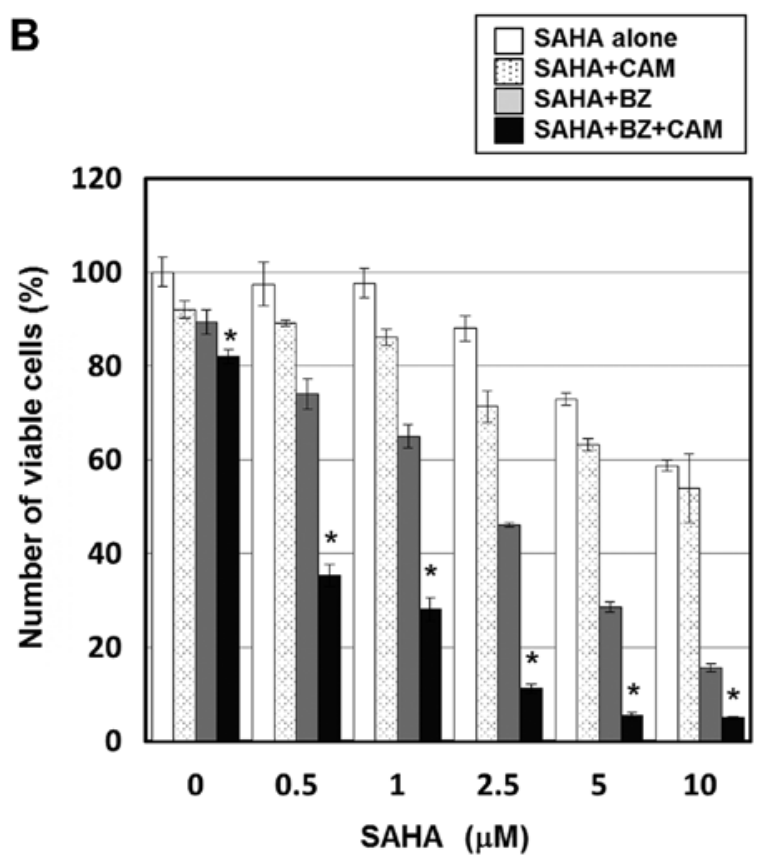

D
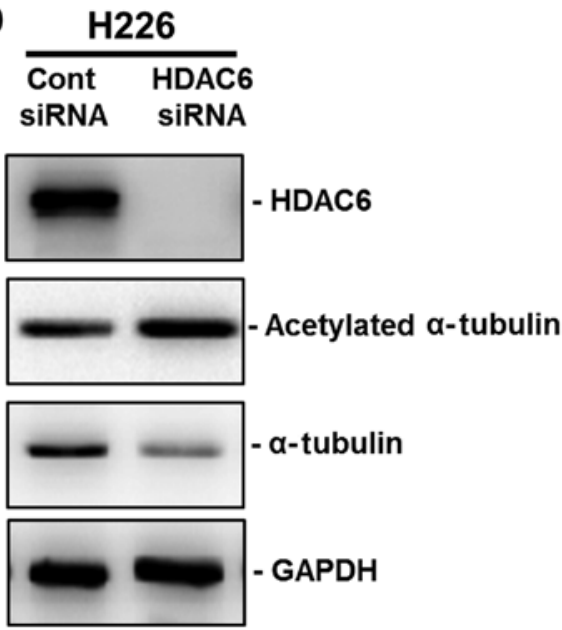

Figure 4. Assessment of aggresome formation by immunocytochemistry using anti-vimentin monoclonal antibody (mAb). (A) RPMI-8226 cells were treated with either bortezomib (BZ) $(5 \mathrm{nM})$ or clarithromycin (CAM) $(50 \mu \mathrm{g} / \mathrm{ml})$ in the presence/absence of suberoylanilide hydroxamic acid (SAHA) $(0.5 \mu \mathrm{M})$ for $16 \mathrm{~h}$. Immunocytochemistry using anti-vimentin $\mathrm{mAb}$ was performed. 4',6-diamidino-2-phenylindole (DAPI) staining shows the position of nucleus (blue). (B) H226 cells were cultured in the presence of various concentrations of SAHA with/without either BZ (5 nM) or CAM $(50 \mu \mathrm{g} / \mathrm{ml})$ for $48 \mathrm{~h}$. Viable cell numbers were assessed as described in Materials and methods. ${ }^{*} \mathrm{P}<0.05 \mathrm{SAHA} / \mathrm{BZ} / \mathrm{CAM}$ vs. SAHA/BZ and SAHA/CAM. (C) H226 cells were treated with either BZ (5 nM) or CAM $(50 \mu \mathrm{g} / \mathrm{ml})$ in the presence/absence of SAHA $(0.5 \mu \mathrm{M})$ for $24 \mathrm{~h}$. Immunocytochemistry using anti-vimentin mAb was performed. (D) H226 cells were pre-treated with histone deacetylase 6 (HDAC6) siRNA or control siRNA for $48 \mathrm{~h}$. Then, the expression levels of HDAC6 and acetylated $\alpha$-tubulin were assessed by immunoblotting. (E) After pre-treatment with siRNA, H226 cells were further exposed to BZ (5 nM) and/or CAM $(50 \mu \mathrm{g} / \mathrm{ml})$ for $24 \mathrm{~h}$. Immunocytochemistry was performed using anti-vimentin $\mathrm{mAb}$. 
$\mathbf{F}$
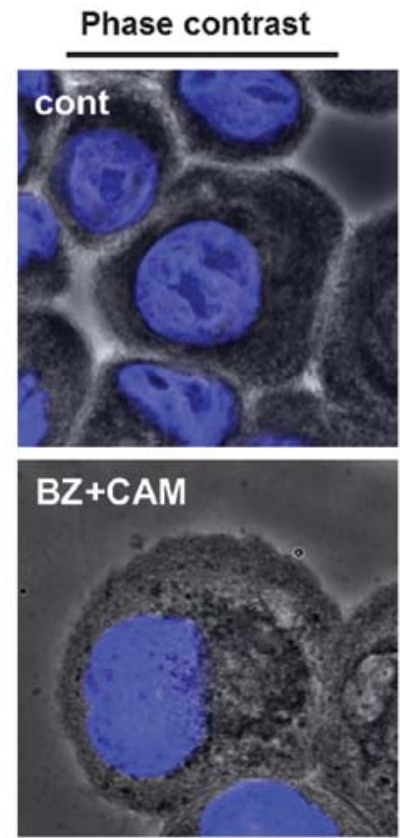

\section{Anti-vimentin $\mathrm{mAb}$}

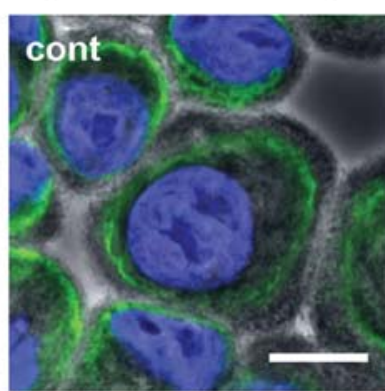

$\mathrm{BZ}+\mathrm{CAM}$

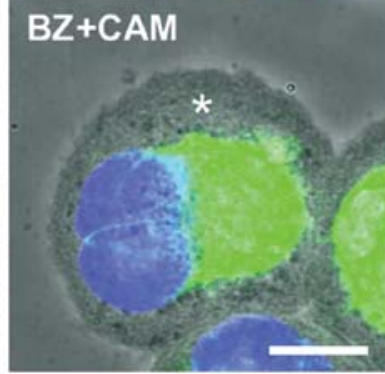

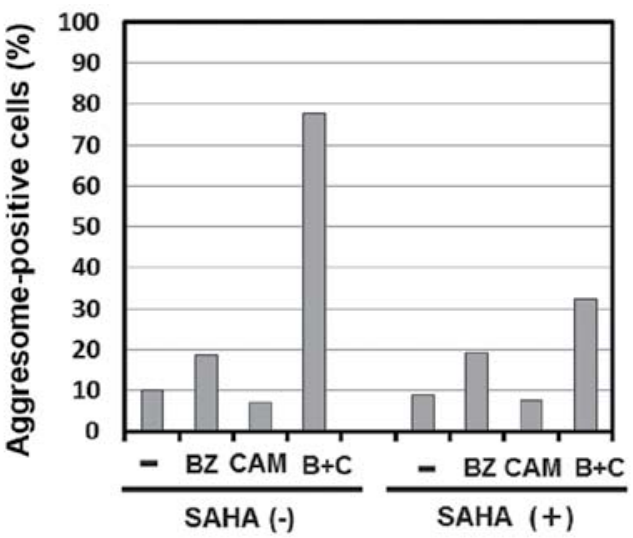

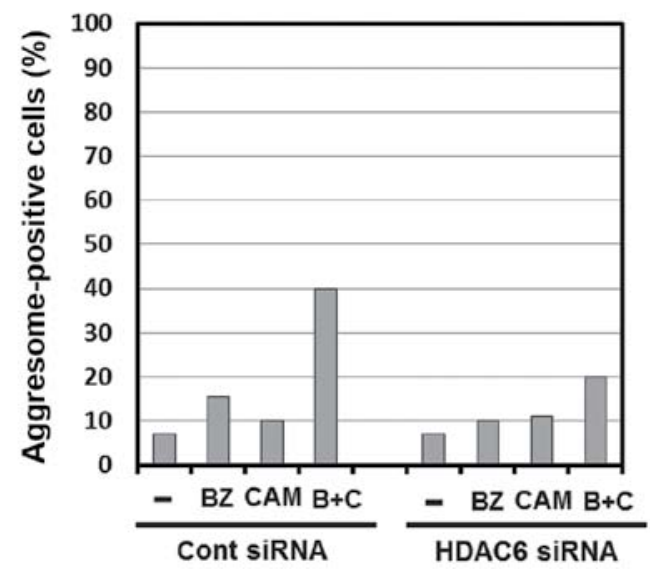

Figure 4. Continued. Assessment of aggresome formation by immunocytochemistry using anti-vimentin monoclonal antibody (mAb). (F) Percentage of 'aggresome-positive' cells in $\mathrm{H} 226$ cells after treatment with either BZ $\pm \mathrm{CAM} \pm \mathrm{SAHA}$ or BZ $\pm \mathrm{CAM}$ pre-treated with HDAC6 siRNA or control siRNA. One hundred cells stained with anti-vimentin $\mathrm{mAb}$ were assessed for their staining pattern. The cells with high dense staining in the perinuclear region (indicated by ${ }^{*}$ in the left lower panel) were defined and plotted as 'aggresome-positive' cells. Each scale bar indicates $10 \mu \mathrm{m}$.

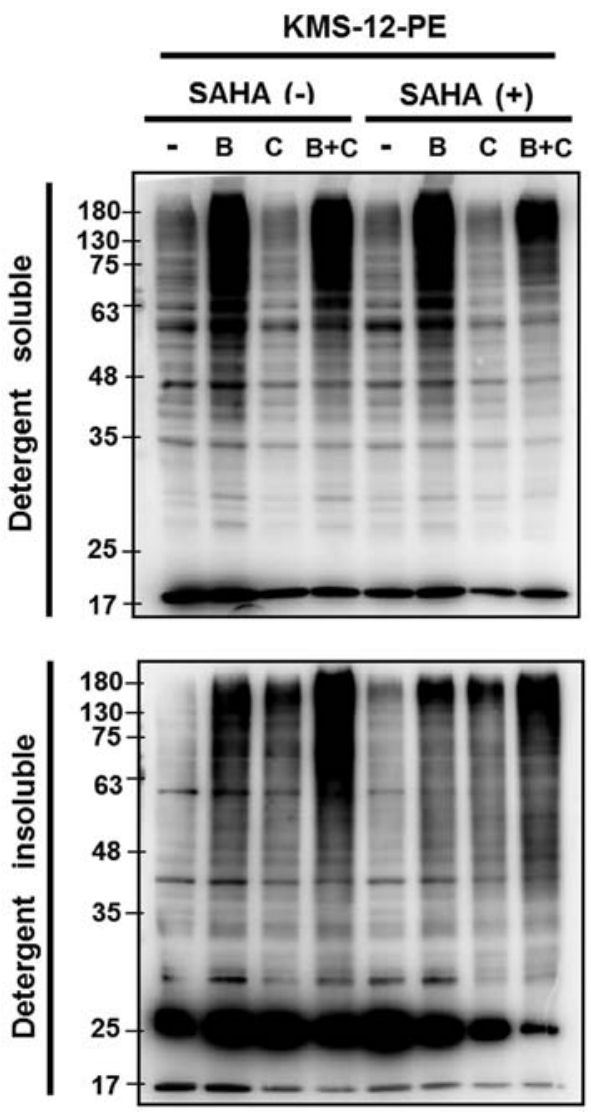

Triton X-100, and cellular proteins were subsequently fractionated into the detergent-soluble and the -insoluble fraction. The detergent-insoluble fraction (cell pellets) was sonicated for $30 \mathrm{sec}$ in the presence of $2 \%$ SDS and boiled for $5 \mathrm{~min}$. Thereafter, the samples were loaded on the gels, separated by $11.25 \%$ SDS-PAGE, and immunoblotted with anti-Ub Ab. The samples derived from the detergent-soluble fractions were processed similarly. As shown in Fig. 5, the increased intracellular polyubiquitinated proteins were detectable after treatment with $\mathrm{BZ}$ and $\mathrm{BZ} / \mathrm{CAM}$, but not with $\mathrm{CAM}$ or SAHA in the detergent-soluble fraction (upper panel). In the detergent-insoluble fraction (lower panel) at higher molecular weights, prominent polyubiquitinated proteins were detected as aggresome contents in the cells treated with BZ/CAM.

Figure 5. Assessment of aggresome formation by immunoblotting using anti-ubiquitin (Ub) monoclonal antibody (mAb). KMS-12-PE cells were treated with either bortezomib (B) $(5 \mathrm{nM})$ or clarithromycin (C) $(50 \mu \mathrm{g} / \mathrm{ml})$ in the presence/absence of suberoylanilide hydroxamic acid (SAHA) $(0.5 \mu \mathrm{M})$ for $16 \mathrm{~h}$. Then cellular proteins were lysed with lysis buffer containing $2 \%$ Triton X-100 for $30 \mathrm{~min}$ at $0^{\circ} \mathrm{C}$ and followed by centrifugation at $14,000 \times \mathrm{g}$ for $30 \mathrm{~min}$. The supernatants were used for 'soluble fractions'. Cell pellets were resuspended in buffer containing $2 \%$ sodium dodecyl sulfate (SDS) and sonicated for $30 \mathrm{sec}$ and further used as 'insoluble fraction'. Each fractionated protein was loaded and separated by $11.25 \%$ sodium dodecyl sulfate polyacrylamide gel electrophoresis (SDS-PAGE), and immunoblotted with anti-Ub mAb. 

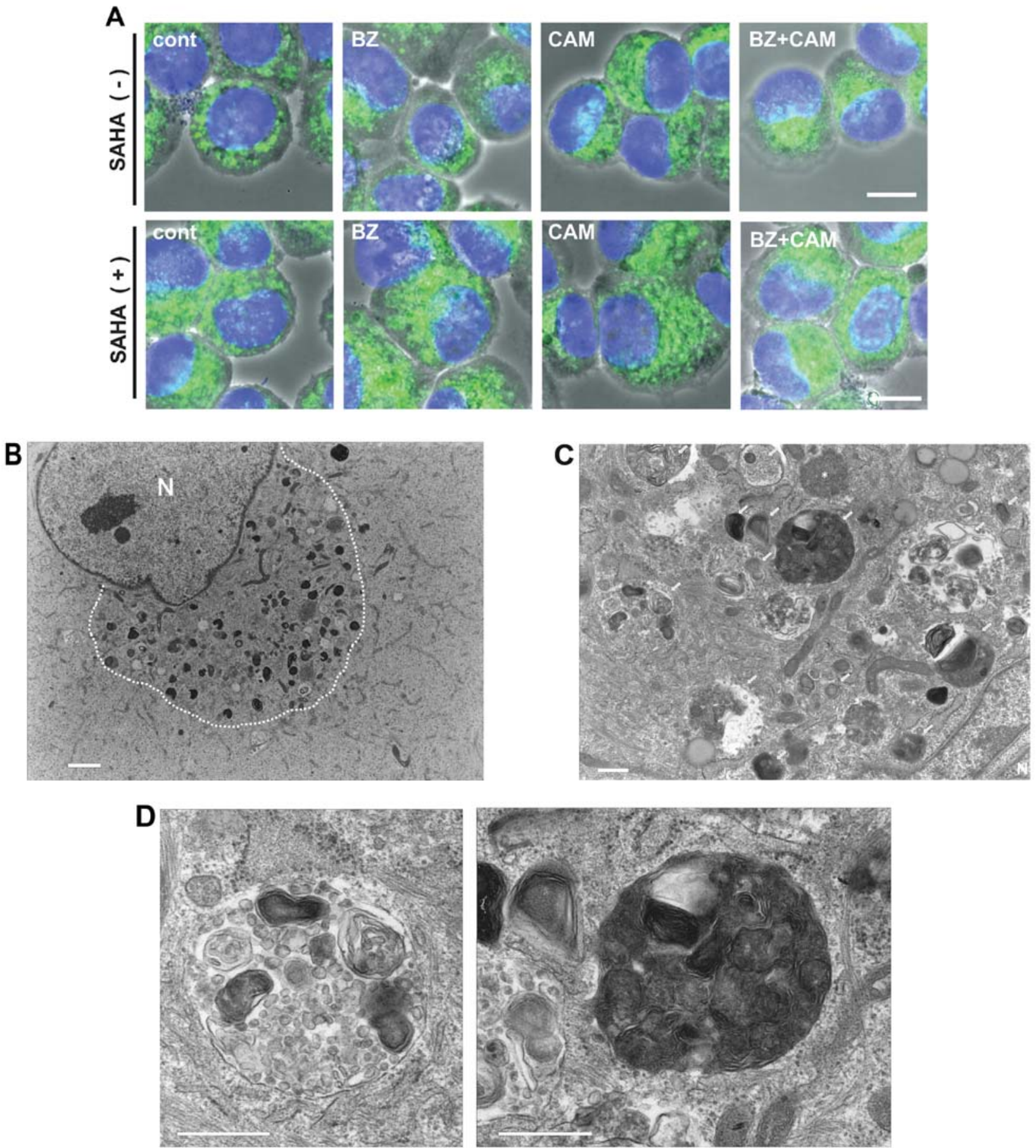

Figure 6. Lysosome positioning after treatment with bortezomib (BZ) and/or clarithromycin (CAM) in the presence or absence of suberoylanilide hydroxamic acid (SAHA) in H226 cells. (A) H226 cells were treated with BZ and/or CAM in the presence or absence of SAHA for 24 h. Then, immunocytochemistry was performed using anti-LAMP-1 monoclonal antibody ( $\mathrm{mAb}$ ) for detecting lysosome positioning. Merged images with phase contrast, 4',6-diamidino-2-phenylindole (DAPI) (blue), and lysosomal-associated membrane protein-1 (LAMP-1) (green). Each scale bar indicates $10 \mu \mathrm{m}$. (B-D) Electron microscopy of H226 cells after treatment with BZ/CAM. (B) The region surrounded by the dashed line shows the clustering of organelles including lysosomes and mitochondria. (C) The arrows indicate autophagosomes and autolysosomes. The asterisk (*) appears to represent a precursor form of aggresome. (D) Clustering autolysosomes/lysosomes might represent 'lysophagy' (30). N, nucleus. Each scale bar indicates (B) $2 \mu \mathrm{m}$ and (C and D) $500 \mathrm{~nm}$.

Treatment with BZ alone also induced accumulation of the detergent-insoluble polyubiquitinated proteins but to a lesser extent than that induced by treatment with BZ/CAM. It was noteworthy that the increased ubiquitinated proteins in the detergent-insoluble fraction were suppressed in the presence of SAHA. This also indicates the inhibition of aggresome in response to SAHA.
Immunocytochemistry using anti-LAMP-1 mAb showed lysosome accumulation in the perinuclear region along with aggresome formation after $\mathrm{BZ} / \mathrm{CAM}$ treatment. As is the case of vimentin-positive aggresome formation, the clustering of LAMP-1-positive dots was suppressed in the presence of SAHA (Fig. 6A). We therefore performed electron microscopy for precise analysis of the cytosolic area strongly stained 
with anti-vimentin Ab. However, contrary to what we had expected, electron microscopy exhibited no typical features of the aggresome structure such as massive accumulation of closely packed electron-dense particles surrounded by a cage of bundles of intermediated filaments in the region of the centrosome, which was first described by Johnston $e t$ al in 1998 (30). Instead, there was the clustering of a number of lysosomes, autophagosomes, autolysosomes, and mitochondria with increased filaments in response to the BZ/CAM treatment (Fig. 6B). A few numbers of unstructured particles with a rather high density, which might be the aggresome precursor, were detectable in this area (Fig. 6C). It was noteworthy that many lysosomal fusions or lysosomal aggregates in various sizes were observed. These might represent the phenomenon termed 'lysophagy' which has recently been reported by others, that is, the lysosome engulfed into an autophagosome or autolysosome (Fig. 6D) (31). In the presence of SAHA, the clustering of these organelles in the perinuclear region was suppressed to some extent along with the decreased filamentous structures. However, an increased number of lysosomes including autolysosomes was still observed compared with the cells treated with either CAM or BZ alone (Fig. 6A).

Concomitant aggresome, proteasome, and autophagy targeting enhanced ER stress loading. Since aggresome is reported to form as an adaption process against overabundant intracellular unfolded or misfolded proteins, we hypothesized that HDAC6 inhibition with SAHA further enhances BZ/CAM-induced ER stress loading to the cells. As we expected, the expression levels of ER stress-related genes including $\mathrm{CHOP}$ were markedly pronounced in the presence of SAHA; the expression ratios of $\mathrm{CHOP}$ to untreated control cells increased to 5-fold by BZ/CAM treatment, and further increased to 25 -fold by BZ/CAM/SAHA treatment in IM-9 cells (Fig. 7A). In H226 cells, similarly to SAHA, HDAC6 siRNA treatment enhanced BZ/CAM-induced CHOP expression compared with the cells treated with control siRNA (Fig. 7B). CHOP is an ER stress-related pro-apoptotic transcription factor which upregulates pro-apoptotic genes (e.g., $B I M, B A X, D R 5)$ and one of the critical molecules playing a role in the induction of apoptosis in response to ER stress $(9,10)$. In addition, enhanced cytotoxicity was shown by a wild-type MEF cell line along with CHOP upregulation by the combination of the three reagents, whereas the $\mathrm{CHOP}^{-/-} \mathrm{MEF}$ cell line derived from a $\mathrm{CHOP}$-deficient mouse almost completely canceled this pronounced cytotoxicity (Fig. 7C). Therefore, the pronounced cytotoxicity and apoptosis in response to the SAHA/BZ/CAM combination treatment shown in Fig. 2A is strongly suggested to be due to the upregulation of CHOP in response to ER stress loading.

\section{Discussion}

We showed that ER stress-mediated apoptosis in MM cells was potently enhanced by the simultaneous targeting of aggresome formation, proteasome, and the autophagy-lysosome system. Concomitant SAHA, BZ, and CAM treatment markedly enhanced CHOP induction via ER stress loading in MM cells (Fig. 7A), whereas a $\mathrm{CHOP}^{-/ /}$MEF cell line nearly completely cancelled the pronounced cytotoxicity in response to the SAHA/BZ/CAM combination treatment (Fig. 7C). These results strongly suggest that CHOP upregulation appears to be involved, at least in part, in the pronounced cytotoxicity shown in Fig. 2A. Our recent report also showed that combined treatment with SAHA/BZ/CAM exhibited potent cytotoxicity along with upregulation of CHOP in breast cancer cell lines (24). Since this enhanced cytotoxicity by ER stress loading is associated with inhibition of the BZ/CAM-induced aggresome formation (Figs. 4 and 5), induction of aggresome itself appears to function as cytoprotective against intracellular overloading of unfolded proteins as previously demonstrated in various neurodegenerative disorders $(32,33)$.

In this study, we intentionally attempted to use the clinically approved drugs for blocking aggresome formation and the proteolytic pathways. Although exhibiting a potent inhibitory effect on HDAC6, SAHA (vorinostat) is a pan-HDAC inhibitor; therefore, the inhibitory effects on other HDACs cannot be completely excluded for the pronounced ER stress loading and apoptosis induction shown in Figs. 2A and 7A. However, tubacin, which is a specific inhibitor of HDAC6, also exhibited pronounced cytotoxicity (Fig. 3B), and knockdown of HDAC6 superimposed the effects of SAHA, such as suppression of vimentin-positive aggresome formation induced by BZ/CAM treatment (Fig. 4C and E), and further enhanced the ER stress-related gene upregulation along with the enhanced BZ/CAM treatment cytotoxicity (Figs. 3A and 7B). Therefore, HDAC6 appears to be a pivotal target for efficient ER stress loading under proteasome/autophagy inhibition. From the standpoint of ER stress loading, intracellular active protein synthesis appears to be theoretically an important factor for determining sensitivity. MM cell sensitivity to BZ/SAHA-induced cell death is regulated by Myc (34). Intracellular ER content, protein synthesis rates, percentage of aggresome-positive cells, and sensitivity to BZ/SAHA-induced cell death directly correlated with Myc expression. We previously reported that cycloheximide suppressed BZ/CAM-induced ER stress loading and cytotoxicity in MM cells (16). Thus, the potent effects shown in this study should depend in part on de novo protein synthesis. This may explain some differences among the MM cell lines, H226 cells, and MEF cell lines in terms of cytotoxicity and ER stress loading in response to these reagents shown in this study.

Along with vimentin-positive aggresome formation in the perinuclear region, Fig. 6 shows a number of autophagosomes and autolysosomes after BZ/CAM treatment. Electron microscopy demonstrated the clustering of autolysosomes in this area. These findings are in agreement with a recent study demonstrating that proteasome inhibition causes the formation of a zone around the centrosome where microtubular transport of lysosomes is suppressed, resulting in lysosome entrapment and accumulation (35). Interestingly, the authors of the previous study reported that the microtubule-dependent transport of other organelles, including autophagosomes, mitochondria and endosomes, is also blocked in this entrapment zone. Therefore, the perinuclear region stained with anti-vimentin $\mathrm{Ab}$, where autolysosomes clustered along with the increased number 
A
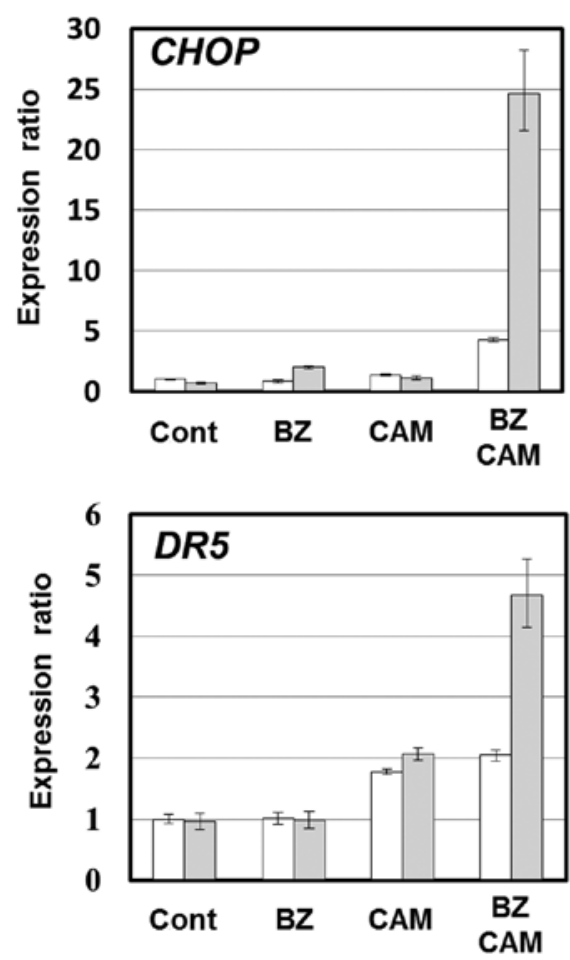

B
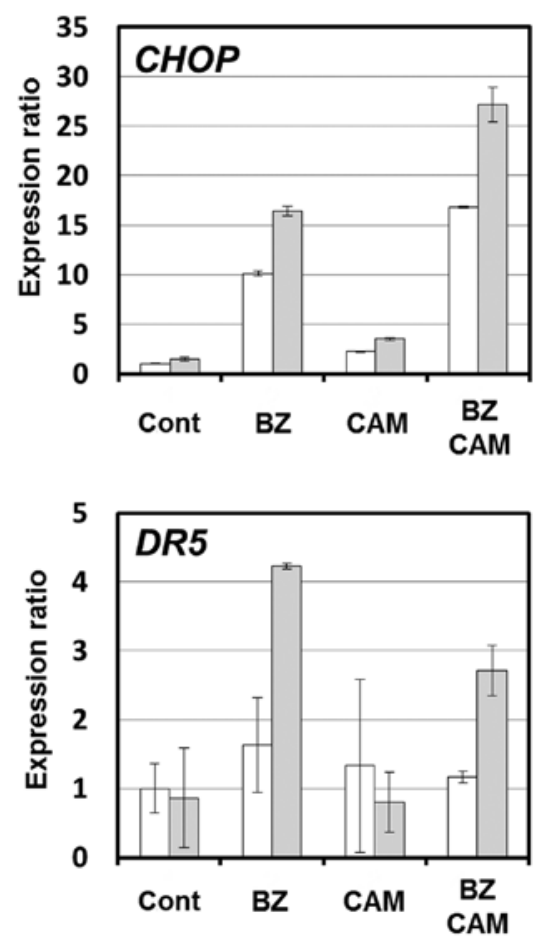
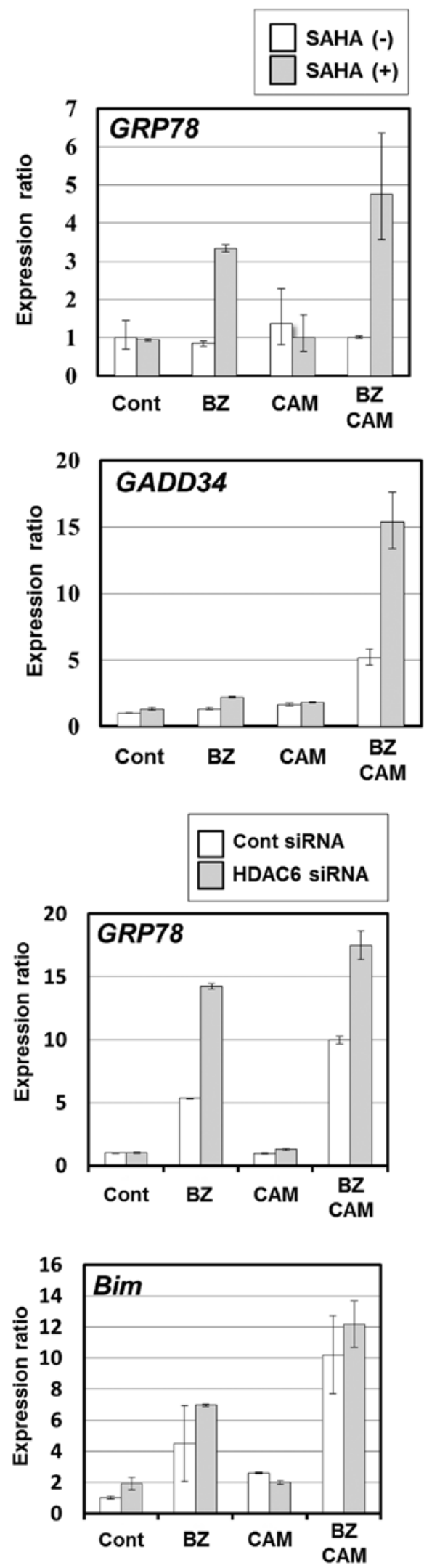

Figure 7. Endoplasmic reticulum (ER) stress loading after combined treatment with suberoylanilide hydroxamic acid (SAHA), bortezomib (BZ), and clarithromycin (CAM). (A) IM-9 cells were treated with BZ $(5 \mathrm{nM})$ and/or CAM $(50 \mu \mathrm{g} / \mathrm{ml})$ in the presence or absence of SAHA $(1 \mu \mathrm{M})$ for $16 \mathrm{~h}$. Gene expressions related to ER stress were assessed by real-time polymerase chain reaction (PCR). (B) Effects of histone deacetylase 6 (HDAC6) siRNA on ER stress-related gene expressions in $\mathrm{H} 226$ cells.

of mitochondria, may in part overlap to 'the entrapment zone' (Figs. 4E and 6B) (35). It is also suggested that, upon proteasome inhibition, the targeting of aggregated proteins to aggresome is coordinated with lysosome positioning around 

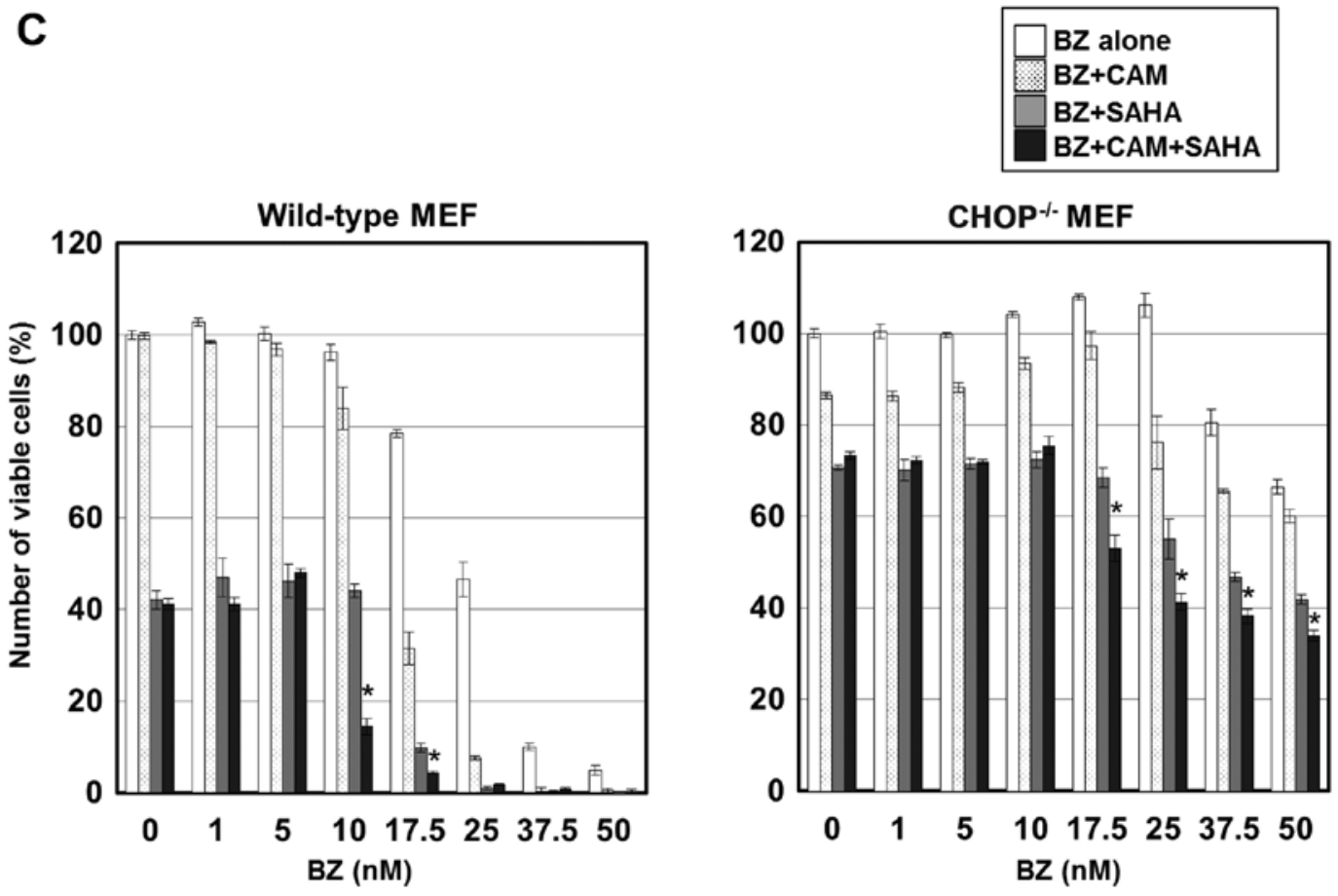

Figure 7. Continued. Endoplasmic reticulum (ER) stress loading after combined treatment with suberoylanilide hydroxamic acid (SAHA), bortezomib (BZ),

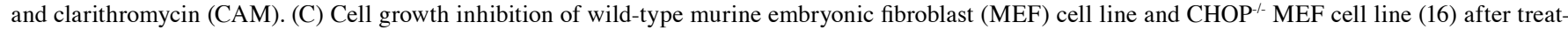
ment with BZ in the presence/absence of SAHA $(2.5 \mu \mathrm{M})$ and/or CAM $(50 \mu \mathrm{g} / \mathrm{ml})$ for $48 \mathrm{~h}$. Viable cell numbers were assessed as described in Materials and methods. ${ }^{*} \mathrm{P}<0.05 \mathrm{BZ} / \mathrm{CAM} / \mathrm{SAHA}$ vs. BZ/SAHA and BZ/CAM.

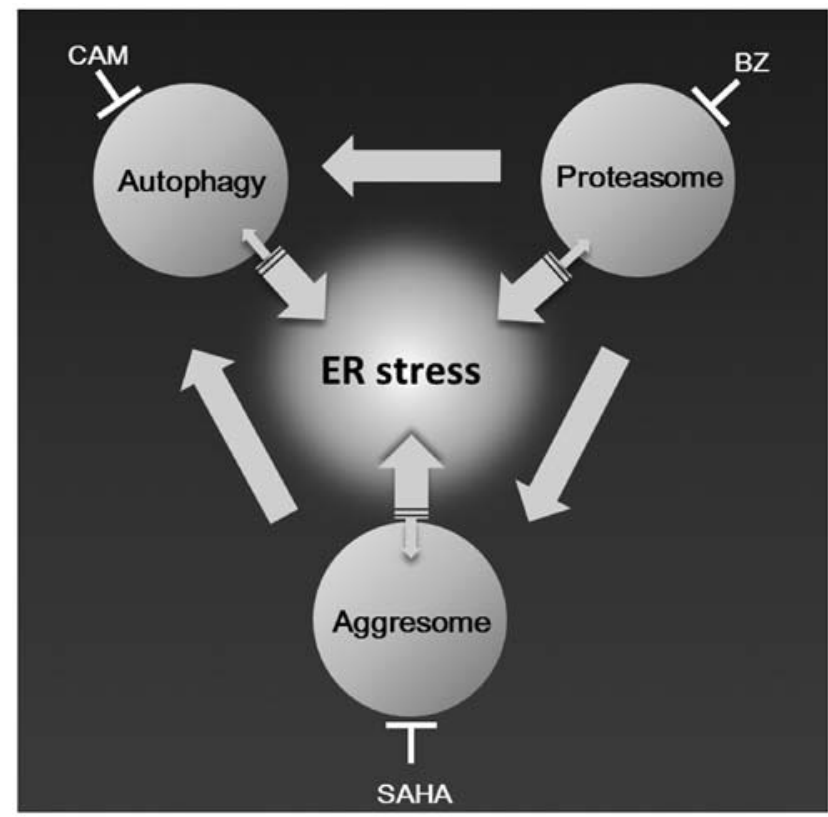

Figure 8. Proposed scheme for intracellular networks of unfolded protein processing and endoplasmic reticulum (ER) stress loading via systematic blockage of proteolytic pathways and aggresome formation.

the body to facilitate degradation via autophagy. However, our data showed that lysosomal clustering including autolysosome further became prominent by the combined treatment with $\mathrm{CAM} / \mathrm{BZ}$ compared with that by the $\mathrm{BZ}$ treatment alone (Fig. 4F). Indeed, the addition of SAHA in the CAM/BZ culture medium suppressed lysosomal clustering in the perinuclear region; however, an increased number of cytosolic autolysosomes were still detectable (data not shown). This was possibly because of the suppression of lysosomal clearance upon autophagy inhibition by CAM. We and others have reported that macrolide antibiotics inhibit autophagy flux, although the precise molecular mechanism still remains to be clarified $(16,17,36)$. The macrolide antibiotic BAF, which is a well-used autophagy inhibitor for in vitro experiments, was initially evaluated for its selective inhibition of a proton-pumping V-ATPase (37). BAF induces the disruption of vesicular proton gradients and raises the $\mathrm{pH}$ of acidic vesicles at nanomolar concentrations. This disruption of vesicular acidification in response to BAF appears to prevent autophagosome fusion with lysosomes, resulting in the inhibition of autophagy. It was also reported that treatment with AZM increased lysosomal $\mathrm{pH}$ in macrophages, which may lead to the inhibition of lysosomal hydrolases having an optimal low $\mathrm{pH}$ for their enzymatic activities (38). In this context, if CAM exerts the same effect on lysosomes, accumulation of autolysosomes by the suppression of lysosomal clearance itself appears to make 'lysophagy' apparent under proteasome inhibition. A similar phenomenon regarding the selective sequestration of damaged lysosome by autophagy was previously reported, in which under the condition of lysosomal damage induced by silica, monosodium urate, and a lysomotropic reagent, autophagy loss results in the in vitro inhibition of lysosomal biogenesis and the in vivo deterioration of acute kidney injury (39). Identification of the target molecules of macrolide antibiotics involved in autophagy flux inhibition, as well as the molecular mechanism of lysophagy including the recognition process of impaired lysosomes appears to be important issues that need to be clarified. 
Taking our data and accumulating lines of evidence together, we can draw an integrated network scheme among aggresome, proteasome, and autophagy as shown in Fig. $8(9,10,13,14)$. Upon inhibition of proteasome and autophagy, overabundant ubiquitinated protein aggregates are transported to MTOC to form aggresomes. Under this condition, further inhibition of aggresome formation most efficiently induces ER stress loading in cells with a high protein synthesis rate such as MM cells. Thus, by combining clinically available drugs such as vorinostat, BZ, and CAM, this systematic strategy for blocking the processing of intracellular unfolded proteins appears to be applicable to refractory/relapsed MM patients.

\section{Acknowledgements}

The authors are indebted to Dr Edward F. Barroga, Associate Professor and Senior Medical Editor of the Department of International Medical Communications of Tokyo Medical University for the editorial review of the report. This study was supported by funds of a Grant-in-Aid for Scientific Research (C) (no. 26460478) and MEXT-Supported Program for the Strategic Research Foundation at Private Universities (S1411011, 2014-2018) from the Ministry of Education, Culture, Sports, Science and Technology of Japan, a Grant-in-Aid from Tokyo Medical University Cancer Research, and a Grant-in-Aid from Taisho Toyama Pharmaceutical Co., Ltd. (Tokyo, Japan) to K.M.; and a Grant-in-Aid for Young Scientist (B) from the Ministry of Education, Culture, Sports, Science and Technology of Japan (no. 25860398) and a Research Grant from Tokyo Medical University to S.M.

\section{References}

1. Richardson PG, Barlogie B, Berenson J, et al: A phase 2 study of bortezomib in relapsed, refractory myeloma. N Engl J Med 348: 2609-2617, 2003.

2. San Miguel JF, Schlag R, Khuageva NK, et al: Bortezomib plus melphalan and prednisone for initial treatment of multiple myeloma. N Engl J Med 359: 906-917, 2008.

3. Richardson PG, Xie W, Jagannath S, et al: A phase 2 trial of lenalidomide, bortezomib, and dexamethasone in patients with relapsed and relapsed/refractory myeloma. Blood 123: 1461-1469, 2014

4. Siegel DS, Martin T, Wang M, et al: A phase 2 study of single-agent carfilzomib (PX-171-003-A1) in patients with relapsed and refractory multiple myeloma. Blood 120: 2817-2825, 2012.

5. Dimopoulos M, Siegel DS, Lonial S, et al: Vorinostat or placebo in combination with bortezomib in patients with multiple myeloma (VANTAGE 088): a multicentre, randomised, double-blind study. Lancet Oncol 14: 1129-1140, 2013.

6. Orlowski RZ: Novel agents for multiple myeloma to overcome resistance in phase III clinical trials. Semin Oncol 40: 634-651, 2013.

7. Martinon F: Targeting endoplasmic reticulum signaling pathways in cancer. Acta Oncol 51: 822-830, 2012.

8. Hetz C, Chevet E and Harding HP: Targeting the unfolded protein response in disease. Nat Rev Drug Discov 12: 703-719, 2013.

9. Tabas I and Ron D: Integrating the mechanisms of apoptosis induced by endoplasmic reticulum stress. Nat Cell Biol 13: 184-190, 2011.

10. Verfaillie T, Salazar M, Velasco G and Agostinis P: Linking ER stress to autophagy: potential implications for cancer therapy. Int J Cell Biol 2010: 930509, 2010.

11. Mizushima N, Levine B, Cuervo AM and Klionsky DJ: Autophagy fights disease through cellular self-digestion. Nature 451 1069-1075, 2008.
12. Mizushima N: Autophagy in protein and organelle turnover. Cold Spring Harb Symp Quant Biol 76: 397-402, 2011.

13. Kirkin V, McEwan DG, Novak I and Dikic I: A role for ubiquitin in selective autophagy. Mol Cell 34: 259-269, 2009.

14. Korolchuk VI, Menzies FM and Rubinsztein DC: Mechanisms of cross-talk between the ubiquitin-proteasome and autophagy-lysosome systems. FEBS Lett 584: 1393-1398, 2010.

15. Kawaguchi T, Miyazawa K, Moriya S, Ohtomo T, Che XF, Naito $M$, Itoh $M$ and Tomoda $A$ : Combined treatment with bortezomib plus bafilomycin $\mathrm{A}_{1}$ enhances the cytocidal effect and induces endoplasmic reticulum stress in U266 myeloma cells: Crosstalk among proteasome, autophagy-lysosome and ER stress. Int J Oncol 38: 643-654, 2011.

16. Moriya S, Che XF, Komatsu S, Abe A, Kawaguchi T, Gotoh A, Inazu M, Tomoda A and Miyazawa K: Macrolide antibiotics block autophagy flux and sensitize to bortezomib via endoplasmic reticulum stress-mediated CHOP induction in myeloma cells. Int J Oncol 42: 1541-1550, 2013.

17. Komatsu S, Miyazawa K, Moriya S, Takase A, Naito M, Inazu M, Kohno N, Itoh $\mathrm{M}$ and Tomoda A: Clarithromycin enhances bortezomib-induced cytotoxicity via endoplasmic reticulum stress-mediated CHOP (GADD153) induction and autophagy in breast cancer cells. Int J Oncol 40: 1029-1039, 2012.

18. Simms-Waldrip T, Rodriguez-Gonzalez A, Lin T, Ikeda AK, $\mathrm{Fu} \mathrm{C}$ and Sakamoto KM: The aggresome pathway as a target for therapy in hematologic malignancies. Mol Genet Metab 94: 283-286, 2008.

19. Kawaguchi Y, Kovacs JJ, McLaurin A, Vance JM, Ito A and Yao TP: The deacetylase HDAC6 regulates aggresome formation and cell viability in response to misfolded protein stress. Cell 115: 727-738, 2003.

20. Ouyang H, Ali YO, Ravichandran M, Dong A, Qiu W, MacKenzie F, Dhe-Paganon S, Arrowsmith $\mathrm{CH}$ and Zhai RG: Protein aggregates are recruited to aggresome by histone deacetylase 6 via unanchored ubiquitin C termini. J Biol Chem 287: 2317-2327, 2012.

21. Lee JY, Koga H, Kawaguchi Y, et al: HDAC6 controls autophagosome maturation essential for ubiquitin-selective quality-control autophagy. EMBO J 29: 969-980, 2010.

22. Fusco C, Micale L, Egorov M, et al: The E3-ubiquitin ligase TRIM50 interacts with HDAC6 and p62, and promotes the sequestration and clearance of ubiquitinated proteins into the aggresome. PLoS One 7: e40440, 2012.

23. Yan J, Seibenhener ML, Calderilla-Barbosa L, Diaz-Meco MT, Moscat J, Jiang J, Wooten MW and Wooten MC: SQSTM1/p62 interacts with HDAC6 and regulates deacetylase activity. PLoS One 8: e76016, 2013.

24. Komatsu S, Moriya S, Che XF, Yokoyama T, Kohno N and Miyazawa K: Combined treatment with SAHA, bortezomib, and clarithromycin for concomitant targeting of aggresome formation and intracellular proteolytic pathways enhances ER stress-mediated cell death in breast cancer cells. Biochem Biophys Res Commun 437: 41-47, 2013.

25. Olsen EA, Kim YH, Kuzel TM, et al: Phase IIb multicenter trial of vorinostat in patients with persistent, progressive, or treatment refractory cutaneous T-cell lymphoma. J Clin Oncol 25: 3109-3115, 2007.

26. Kavanaugh SM, White LA and Kolesar JM: Vorinostat: a novel therapy for the treatment of cutaneous T-cell lymphoma. Am J Health Syst Pharm 67: 793-797, 2010.

27. Hubbert C, Guardiola A, Shao R, Kawaguchi Y, Ito A, Nixon A, Yoshida M, Wang XF and Yao TP: HDAC6 is a microtubule-associated deacetylase. Nature 417: 455-458, 2002.

28. Haggarty SJ, Koeller KM, Wong JC, Grozinger CM and Schreiber SL: Domain-selective small-molecule inhibitor of histone deacetylase 6 (HDAC6)-mediated tubulin deacetylation. Proc Natl Acad Sci USA 100: 4389-4394, 2003.

29. García-Mata R, Bebök Z, Sorscher EJ and Sztul ES: Characterization and dynamics of aggresome formation by a cytosolic GFP-chimera. J Cell Biol 146: 1239-1254, 1999.

30. Johnston JA, Ward CL and Kopito RR: Aggresomes: a cellular response to misfolded proteins. J Cell Biol 143: 1883-1898, 1998.

31. Hung YH, Chen LM, Yang JY and Yang WY: Spatiotemporally controlled induction of autophagy-mediated lysosome turnover. Nat Commun 4: 2111, 2013

32. Richter-Landsberg C and Leyk J: Inclusion body formation, macroautophagy, and the role of HDAC6 in neurodegeneration. Acta Neuropathol 126: 793-807, 2013.

33. Hol EM, Fischer DF, Ovaa H and Scheper W: Ubiquitin proteasome system as a pharmacological target in neurodegeneration. Expert Rev Neurother 6: 1337-1347, 2006. 
34. Nawrocki ST, Carew JS, Maclean KH, Courage JF, Huang P, Houghton JA, Cleveland JL, Giles FJ and McConkey DJ: Myc regulates aggresome formation, the induction of Noxa, and apoptosis in response to the combination of bortezomib and SAHA. Blood 112: 2917-2926, 2008.

35. Zaarur N, Meriin AB, Bejarano E, Xu X, Gabai VL, Cuervo AM and Sherman MY: Proteasome failure promotes positioning of lysosomes around the aggresome via local block of microtubule-dependent transport. Mol Cell Biol 34: 1336-1348, 2014.

36. Nakamura M, Kikukawa $Y$, Takeya M, Mitsuya $H$ and Hata $H$ : Clarithromycin attenuates autophagy in myeloma cells. Int J Oncol 37: 815-820, 2010.
37. Yamamoto A, Tagawa Y, Yoshimori T, Moriyama Y, Masaki R and Tashiro Y: Bafilomycin $\mathrm{A}_{1}$ prevents maturation of autophagic vacuoles by inhibiting fusion between autophagosomes and lysosomes in rat hepatoma cell line, H-4-II-E cells. Cell Struct Funct 23: 33-42, 1998.

38. Renna M, Schaffner C, Brown K, et al: Azithromycin blocks autophagy and may predispose cystic fibrosis patients to mycobacterial infection. J Clin Invest 121: 3554-3563, 2011.

39. Maejima I, Takahashi A, Omori H, et al: Autophagy sequesters damaged lysosomes to control lysosomal biogenesis and kidney injury. EMBO J 32: 2336-2347, 2013. 